\title{
A robust coupled model for solute transport driven by severe flow conditions
}

\author{
Lili Zhang $^{\mathrm{a}, \mathrm{b}}$, Qiuhua Liang ${ }^{\mathrm{a}, \mathrm{b}, *}$, Yueling Wang ${ }^{\mathrm{c}}$, Junxian Yin ${ }^{\mathrm{a}}$ \\ ${ }^{a}$ State Key Laboratory of Simulation and Regulation of Water Cycle in River Bain, China Institute of Water Resources and Hydropower Research, Beijing 100038, \\ China \\ ${ }^{\mathrm{b}}$ School of Civil Engineering and Geosciences, Newcastle University, Newcastle upon Tyne NE1 7RU England, UK \\ ${ }^{\mathrm{c}}$ Key Laboratory of Water Cycle and Related Land Surface Processes, Institute of Geographic Sciences and Natural Resources Research, Chinese Academy of \\ Sciences, 100101, China
}

Received 30 August 2013; revised 2 April 2014; accepted 3 April 2014

Available online 18 November 2014

\begin{abstract}
This paper introduces a computationally efficient model that solves a $4 \times 4$ matrix form of the hyperbolic conservation laws consisting of the $2 \mathrm{D}$ shallow water and advection-diffusion equations. The model allows automatic shock-capturing due to the implementation of a finite volume Godunov-type scheme featured with an HLLC approximate Riemann solver. The numerical scheme is also able to provide well-balanced solutions and maintain non-negative water depth and solute concentration for applications involving wetting and drying over complex domain topographies. Implemented on a simplified adaptive grid system, the model can save 3-17 times of computational cost without compromising solution accuracy for those simulations with predominant localised complex hydrodynamic or flow features, as demonstrated by the numerical experiments. Therefore, the current model provides a potential tool for efficient simulation of large-scale solute transport as well as flow hydrodynamics during a highly transient flood event caused by dam failure or flash flooding.

(C) 2014 International Association for Hydro-environment Engineering and Research, Asia Pacific Division. Published by Elsevier B.V. All rights reserved.
\end{abstract}

Keywords: Adaptive mesh simulation; Advection and diffusion; Shallow water equations; Godunov-type scheme; Wetting and drying; Complex topography

\section{Introduction}

Recently in 2012, a number of severe flash flood events have been reported in different places around the globe. For example, in January 2012, with nearly $200 \mathrm{~mm}$ of rain recorded in $24 \mathrm{~h}$, severe flash flooding occurred in different parts of Brisbane in Australia, not long after the massive 2011 flood had claimed 38 lives. In July 2012, a devastating flash flood killed 144 people in the Krasnodar Region in Russia, and Beijing in China, Uttarakhard in India and Manila in the Philippines have also since been struck. Most commonly associated with torrential rainfall, these flash floods are

\footnotetext{
* Corresponding author. State Key Laboratory of Simulation and Regulation of Water Cycle in River Bain, China Institute of Water Resources and Hydropower Research, Beijing, 100038, China.

E-mail address: Qiuhua.Liang@ncl.ac.uk (Q. Liang).
}

characterised by a sudden rise in the water level in rivers and floodplains and very high flow velocity. Due to their violent and unpredictable nature, flash floods pose a great threat to human lives and property. In a city, this type of flood events may strike and damage water treatment plants or other facilities that have potential to release pollutants. More commonly, water quality problem may be linked to surcharge of sewer systems under the extreme flood conditions that exceed the design capacity. This will affect public health and worsen the already devastated impacts of the flood that have posed on people. In order to facilitate the flood risk management associated with polluted flood water, it is important to have a robust model that can predict the fate of the point-source pollutant/solute that is transported by flood waves. In this context, a model should 1) allow shock-capturing as to accurately describe the violent flow hydrodynamics induced by flash floods including rapid wetting and drying processes, 2) 
be able to represent the complex topographic features in a realworld floodplain, especially in a developed urban area, and 3) be very efficient to facilitate risk analysis.

In the last two decades, due the availability of a rich source of high-resolution catchment and floodplain data and the development of computing hardware and modern computational methods, two-dimensional hydraulic flood modelling tools have been widely reported and applied in different types of flood management work. By using different form of the momentum equations, these hydraulic models may be generally classified as dynamic-wave, diffusion-wave and kinematic-wave models. Among them, the diffusion-wave models have gained particular popularity due to the use of substantially simplified governing equations and hence the possibility of increased computational efficiency (e.g. Bates and De Roo, 2000; Bates et al., 2010; Aricò et al., 2011; Wang et al., 2011). Aricò et al. (2011) and several references therein provided a more detailed discussion.

However, in relation to the aforementioned problem of pollutant spreading caused by dam breaks or flash floods, the flow and solute dynamics may be highly complex and involve discontinuous flood waves and contact interfaces over irregular domain topographies. Seeking reliable numerical solution to this type of problems is beyond the capability of the diffusion-wave or other simplified models. This requires a robust modelling tool with shock-capturing capability and it is more appropriate to solve simultaneously the shallow water and pollutant transport equations in an integrated form (Toro, 2001; Liang et al., 2003). The resulting integrated governing equations represent a system of hyperbolic conservation laws that possess the same mathematical property as the original shallow water equations. Therefore the shock-capturing Godunov-type numerical schemes that have been widely applied to solve the shallow water equations can be directly adopted to resolve the integrated conservation laws (Toro, 2001). A number of models of this kind have been reported in literature in recent years and most of them focus on seeking stable and well-balanced numerical solutions in the context a shockcapturing Godunov-type scheme for applications related to wetting and drying over rough terrains (Murillo et al., 2005, 2006; Benkhaldoun et al., 2007; Petti and Bosa, 2007; Murillo et al., 2008, 2009; Liang, 2010b; Murillo and García-Navarro, 2011; Cea and Vázquez-Cendón, 2012). Among these, the well-balanced finite volume Godunov-type scheme presented recently by Liang (2010b) was incorporated with a non-negative reconstruction technique and so is more suitable for practical simulations that involve wetting and drying over complex domain topographies. However, the model was originally developed on uniform Cartesian grids and may be computationally highly demanding in case of large-scale high-resolution simulations.

Allowing the density of the underlying computational mesh to be adjusted according to the flow solution during a simulation, adaptive mesh refinement (AMR) provides an effective way to improve numerical efficiency without compromising too much the solution accuracy. A number of different adaptive grid systems have been reported in literature to achieve
AMR simulations. For example, an effective block-structured AMR was introduced by Berger and Oliger (1984) and Berger and Colella (1989), which was later implemented in Berger et al. (2011) for solving the shallow water equations and subsequently used by George (2011) for dam-break simulations. Due to their flexibility in performing grid adaptation, quadtree grids have been widely used to solve the shallow water equations for different applications, e.g. (Roger et al., 2001; Liang et al., 2003; Liang and Borthwick, 2009; Popinet, 2011). In order to further simplify the quadtree data structure for storing grid information, Liang (2012) recently proposed a simplified adaptive grid systems for solving partial differential equations. Starting from a non-uniform but locally structured grid, the simplified adaptive grid system allows dynamic grid adaptation by simply altering the subdivision level of a coarse background cell according to certain criteria. No data structure is required for neighbour finding. The new grid systems have been implemented in different numerical schemes (Wang and Liang, 2011; Kesserwani and Liang, 2012; Zhang et al., 2013) to solve the shallow water equations for flood simulations.

In this work, the model reported by Liang (2010b) will be incorporated with the simplified adaptive grid system to improve its performance for practical applications. Compared with its uniform grid counterpart, the new adaptive grid based model is able to produce results with similar solution accuracy but at a much lower computational cost. Therefore it has a better potential in large-scale simulations of real-world problems.

\section{Grid generation}

To enable solution adaption, a simulation generally starts from a non-uniform grid. This work proposes a simple grid adaptation strategy realized on a non-uniform Cartesian grid system recently reported by Liang (2011), which can be generated according to the following four steps:

1) Fit the computational domain into a rectangle (or square);

2) Discretize the rectangular domain by a coarse uniform grid, which is referred to as background grid hereafter;

3) Check each cell on the background grid and subdivide it by simply allocating specific subdivision levels according to certain criteria;

4) Regularize the grid to ensure that there is no computational cell having a neighbour more than two times bigger or smaller and this is called the 2:1 condition.

Fig. 1 shows a typical configuration of a final regularized grid, where the background cells $(I-1, J),(I, J)$ and $(I+1$, $J$ ) respectively have a subdivision level of 0,1 and 2 . On such a grid, the structured property of a uniform grid is locally maintained and the neighbour information is entirely determined by simple algebraic relationships. Therefore, there is no necessity of explicit data structure to store the neighbour information and so it may be referred to as a structured but nonuniform grid (refer to (Liang, 2011) for details on neighbour 


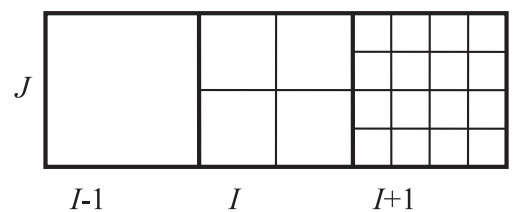

Fig. 1. Typical configuration of a regularized grid.

finding). The grid system facilitates easy dynamical grid adaptation during a flow simulation.

\section{Governing equations}

In this work, the shallow flow hydrodynamics is described by the shallow water equations while the transport of passive solute concentration is governed by the depth-averaged advection-diffusion equation. The two sets of equations may be written in a $4 \times 4$ matrix form as.

$\frac{\partial \mathbf{q}}{\partial t}+\frac{\partial \mathbf{f}}{\partial x}+\frac{\partial \mathbf{g}}{\partial y}=\frac{\partial \mathbf{f}_{d}}{\partial x}+\frac{\partial \mathbf{g}_{d}}{\partial y}+\mathbf{s}$,

where $t$ is the time, $x$ and $y$ are the Cartesian coordinates, $\mathbf{q}$ represents the vector of flow variables, $\mathbf{f}$ and $\mathbf{g}$ are flux vectors in the $x$ and $y$-directions, $\mathbf{f}_{d}$ and $\mathbf{g}_{d}$ contain the diffusion fluxes and $\mathbf{s}$ denotes the vector of source terms. The vectors may be given as follows

$\mathbf{q}=\left[\begin{array}{llll}\eta & q_{x} & q_{y} & q_{c}\end{array}\right]^{\mathrm{T}}$,

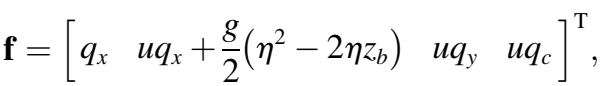

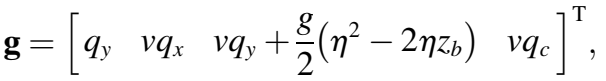

$\mathbf{f}_{d}=\left[\begin{array}{llll}0 & 0 & 0 & h D_{x x} \frac{\partial c}{\partial x}+h D_{x y} \frac{\partial c}{\partial y}\end{array}\right]^{\mathrm{T}}$

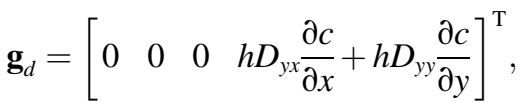

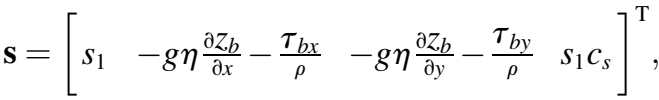

where $\eta$ is the water level, $u$ and $v$ are the $x$ and $y$ components of the depth-averaged velocity, $c$ is the depth-averaged solute concentration, $h=\eta-z_{b}$ gives the total water depth with $z_{b}$ denoting the bottom elevation, $q_{x}(=u h)$ and $q_{y}(=v h)$ are the unit-width discharge in the two Cartesian directions, $q_{c}(=c h)$ is the solute volume per unit area, $g$ represents the acceleration due to gravity, $-\partial z_{b} / \partial x$ and $-\partial z_{b} / \partial y$ are the bed slopes, $\tau_{b x}$ and $\tau_{b y}$ denote the bed friction stresses and are estimated using the following empirical formulae

$\tau_{b x}=\rho C_{f} u \sqrt{u^{2}+v^{2}}$,

and $\tau_{b v}=\rho C_{f} v \sqrt{u^{2}+v^{2}}$

in which $\rho$ is the density of water and $C_{f}=g n^{2} / h^{1 / 3}$ with $n$ being the Manning coefficient. The shallow water Equations (1)-(7) directly employ the water level $\eta$ as a flow variable and therefore ensure well-balanced property of a numerical scheme, i.e. preserve the equilibrium solution of lake at rest (Greenberg and Leroux, 1996), for wet-bed applications. As reported in Liang and Borthwick (2009) and Liang (2010a), the formulation also facilitates a non-negative Godunov-type scheme for dry-bed applications.

\section{Non-negative finite volume Godunov-type scheme}

On the aforementioned structured but non-uniform grids, the integrated formulation of governing Equations (1)-(7) are discretized at each cell using a MUSCL-Hancock finite volume Godunov-type scheme and the interface fluxes are obtained by solving local Riemann problems using an HLLC approximate Riemann solver.

The MUSCL-Hancock method achieves second-order accuracy in both space and time using a two-step approach, i.e. a predictor step and a corrector step. In the predictor step, intermediate values of flow variables are computed over half of a time interval $\Delta t / 2$ using the following formula.

$$
\begin{aligned}
\mathbf{q}_{i}^{k+1 / 2}= & \mathbf{q}_{i}^{k}-\frac{\Delta t}{2 \Delta x_{i}}\left(\mathbf{f}\left(\mathbf{q}^{E}\right)-\mathbf{f}\left(\mathbf{q}^{W}\right)\right)-\frac{\Delta t}{2 \Delta y_{i}}\left(\mathbf{g}\left(\mathbf{q}^{N}\right)-\mathbf{g}\left(\mathbf{q}^{S}\right)\right) \\
& +\frac{\Delta t}{2 \Delta x_{i}}\left(\mathbf{f}_{d}^{E}-\mathbf{f}_{d}^{W}\right)+\frac{\Delta t}{2 \Delta y_{i}}\left(\mathbf{g}_{d}^{N}-\mathbf{g}_{d}^{S}\right)+\frac{\Delta t}{2} \mathbf{s}_{i}^{k},
\end{aligned}
$$

where the superscripts $k$ represents the time level, $E, W, N$ and $S$ indicate the eastern, western, northern and southern interfaces of cell $i$, the subscripts $R$ and $L$ respectively stands for 'Right' and 'Left', $\Delta t$ is the time step and $\Delta x_{i}$ and $\Delta y_{i}$ are the cell dimension in the two Cartesian directions. In order to evaluate the fluxes $\mathbf{f}\left(\mathbf{q}^{E}\right), \mathbf{f}\left(\mathbf{q}^{W}\right), \mathbf{g}\left(\mathbf{q}^{N}\right)$ and $\mathbf{g}\left(\mathbf{q}^{S}\right)$, the quantities $\mathbf{q}^{E}, \mathbf{q}^{W}, \mathbf{q}^{N}$ and $\mathbf{q}^{S}$ must be properly reconstructed at the midpoint of cell interface from the corresponding cell-centre values. A slope limited linear reconstruction, i.e. the MUSCL approach, is adopted in this work, which gives second-order accuracy in space. Taking the eastern interface of cell $i$ as an example,

$\mathbf{q}^{E}=\mathbf{q}_{i}^{k}+\frac{\Delta x_{i}}{2} \nabla \widehat{\mathbf{q}}_{i}$

where $\nabla \widehat{\mathbf{q}}_{i}$ contains the gradients of flow variables that are limited by a TVD Minmod slope limiter defined as

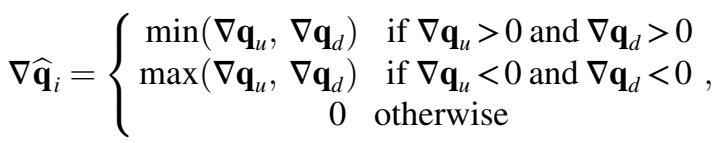

with 
$\nabla \mathbf{q}_{u}=\left(\mathbf{q}_{e}-\mathbf{q}_{i}\right) / \Delta x_{i}$

and

$\nabla \mathbf{q}_{d}=\left(\mathbf{q}_{i}-\mathbf{q}_{w}\right) / \Delta x_{i}$,

where the subscripts $e$ and $w$ refer to the eastern and western neighbours of cell $i$ that are at the same level of subdivision. The face value of water depth $h^{E}$ is also similarly reconstructed, which is then used together with the face value of water level to calculate the bed elevation, i.e.

$z_{b}^{E}=\eta^{E}-h^{E}$,

Then the face values of flow variables and bed elevation (e.g. $\mathbf{q}^{E}, h^{E}$ and $z_{b}^{E}$ ) are directly used to calculate the flux functions at the interfaces, without necessity of solving a Riemann problem.

When a simulation involves wetting and drying, the above linear spatial reconstruction of flow variables reduces to be piecewise with $\nabla \widehat{\mathbf{q}}_{i}=0$ at those cells defining a wet-dry interface, i.e. those wet cells directly neighbouring a dry cell or those dry cells directly adjacent to a wet cell, in order to maintain better numerical stability. Since flux calculation at this step is only related to the values of flow variables at the extremity of the inner interfaces, those cells that are originally dry remain to be dry and no calculation is actually necessary.

The diffusion terms $\mathbf{f}_{d}$ and $\mathbf{g}_{d}$ and the source terms $\mathbf{s}$ (apart from the friction source terms for the shallow water equations which will be mentioned later on) can be simply approximated by central differences due to the use of the pre-balanced shallow water Equations (1)-(7). For example, the $x$-direction slope source term is discretized as $-g \bar{\eta}_{i}^{k \frac{z_{b}^{E}-z_{b}^{W}}{\Delta x_{i}}}$ with $\bar{\eta}_{i}^{k}=\frac{\eta^{E}+\eta^{W}}{2}$.

In the corrector step, the flow variables are updated to a new time step using the following fully conservative timemarching formula.

$$
\begin{aligned}
\mathbf{q}_{i}^{k+1}= & \left.\mathbf{q}_{i}^{k}-\frac{\Delta t}{\Delta x_{i}}\left(\mathbf{f}^{E}-\mathbf{f}^{W}\right)\right)-\frac{\Delta t}{\Delta y_{i}}\left(\mathbf{g}^{N}-\mathbf{g}^{S}\right)+\frac{\Delta t}{\Delta x_{i}}\left(\mathbf{f}_{d}^{E}-\mathbf{f}_{d}^{W}\right) \\
& +\frac{\Delta t}{\Delta y_{i}}\left(\mathbf{g}_{d}^{N}-\mathbf{g}_{d}^{S}\right)+\Delta t \mathbf{s}_{i}^{k+1 / 2}
\end{aligned}
$$

where $\mathbf{f}^{E}, \mathbf{f}^{W}, \mathbf{g}^{N}$ and $\mathbf{g}^{S}$ are the numerical fluxes through the four cell interfaces, defined as a function of the properly reconstructed left and right face values that are also known as Riemann states. For example, with the Riemann states $\tilde{\mathbf{q}}_{L}^{E}$ and $\tilde{\mathbf{q}}_{R}^{E}, \mathbf{f}^{E}$ is defined as

$\mathbf{f}^{E}=f\left(\tilde{\mathbf{q}}_{L}^{E}, \tilde{\mathbf{q}}_{R}^{E}\right)$,

which is mathematically a Riemann problem and its solution provides the fluxes across the eastern interface of cell $i$.

A key step to define the above Riemann problem and subsequently find its solution is to correctly reconstruct the Riemann states. For applications to real-world simulations, the Riemann states must be properly reconstructed to maintain the well-balanced property of the original prebalanced governing equations and meanwhile preserve nonnegativity of the water depth and solute concentration. The method proposed in Liang (2010a) is adopted in this work and modified in the context of the MUSCL-Hancock framework. The face values of flow variables, i.e. at the eastern interface, may be calculated as

$\mathbf{q}_{L}^{\prime E}=\mathbf{q}_{i}^{k+1 / 2}+\frac{\Delta x_{i}}{2} \nabla \widehat{\mathbf{q}}_{i}$

and

$\mathbf{q}_{R}^{\prime E}=\mathbf{q}_{e}^{k+1 / 2}-\frac{\Delta x_{e}}{2} \nabla \widehat{\mathbf{q}}_{e}$.

Herein the limited gradient terms $\nabla \widehat{\mathbf{q}}_{i}$ and $\nabla \widehat{\mathbf{q}}_{e}$ evaluated in the predictor step are reused by following other MUSCLHancock schemes reported in literature (e.g. Liang and Borthwick, 2009). As in the predictor step, the limited gradient terms become zero in those cells defining a wet-dry front. The face values of water depth, $h_{L}^{\prime E}$ and $h_{R}^{\prime E}$, are similarly reconstructed and then used together with the reconstructed water level, $\eta_{L}^{\prime E}$ and $\eta_{R}^{\prime E}$, to calculate the face values of bed elevation.

$z_{b L}^{\prime E}=\eta_{L}^{\prime E}-h_{L}^{\prime E}$

and

$z_{b R}^{\prime E}=\eta_{R}^{\prime E}-h_{R}^{\prime E}$,

which are used to define a single bed function across the cell interface

$\tilde{z}_{b}^{E}=\max \left(z_{b L}^{\prime E}, z_{b R}^{\prime E}\right)$,

from which the non-negative Riemann states of water depth are defined as

$\tilde{h}_{L}^{E}=\max \left(0, \eta_{L}^{\prime E}-\tilde{z}_{b}^{b}\right)$

and

$\tilde{h}_{R}^{E}=\max \left(0, \eta_{R}^{\prime E}-\tilde{z}_{b}^{b}\right)$.

The Riemann states of other flow variables are then reconstructed, e.g. the left states.

$\tilde{\eta}_{L}^{E}=\tilde{h}_{L}^{E}+\tilde{z}_{b}^{E}$,

$\tilde{q}_{x L}^{E}=u_{L}^{\prime E} \tilde{h}_{L}^{E}$

and

$\tilde{q}_{y L}^{E}=v_{L}^{\prime E} \tilde{h}_{L}^{E}$

where $u_{L}^{\prime E}=q_{x L}^{\prime E} / h_{L}^{\prime E}$ (similarly $v_{L}^{\prime E}$ ) is the face value of the velocity, which directly set to zero in a dry cell with water depth smaller than $1.0 \times 10^{-10} \mathrm{~m}$. 


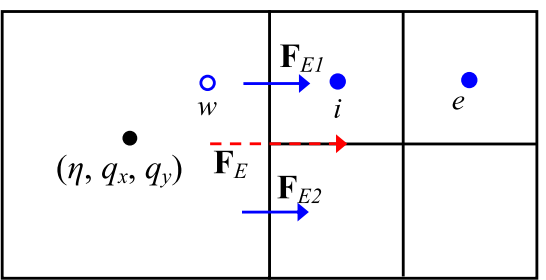

Fig. 2. Flow calculation on the adaptive grid system.

The above reconstruction process may violate the condition of a well-balanced scheme in the case of a wet cell neighbouring a dry cell with bed elevation higher than the water level of the wet cell. A simple local bed modification method as proposed in Liang (2010a) is adopted to restore the wellbalanced solution. Taking the eastern interface of cell $i$ as an example, the difference between the water level at the wet cell and the ground level at the dry neighbour can be found as.

$\Delta z=\max \left(0, \tilde{z}_{b}^{E}-\eta_{L}^{E}\right)$.

Then the bed function and the Riemann states of water level are corrected locally and instantaneously by subtracting $\Delta z$ from their original values, i.e. $\tilde{z}_{b}^{E} \leftarrow \tilde{z}_{b}^{E}-\Delta z, \tilde{\eta}_{L}^{E} \leftarrow \tilde{\eta}_{L}^{E}-\Delta z$ and $\tilde{\eta}_{R}^{E} \leftarrow \tilde{\eta}_{R}^{E}-\Delta z$.
These Riemann states are then employed by an approximate Riemann solver, HLLC approximate Riemann solver herein, to calculate the interface fluxes in (16). As in the predictor step, a compatible discretisation approach must be implemented to evaluate the slope source terms, i.e. $-g \bar{\eta}_{i}^{k+1 / 2} \frac{z_{b}^{E}-\tilde{z}_{b}^{W}}{\Delta x_{i}}$ with $\bar{\eta}_{i}^{k+1 / 2}=\frac{\tilde{\eta}_{L}^{E}+\tilde{\eta}_{R}^{W}}{2}$.

In the current model, as mentioned previously, only the slope source terms of the shallow water equations are included in the above MUSCL-Hancock discretization. The friction source terms are taken into account using a point-implicit scheme as reported in Liang (2010a) to avoid numerical instability that may otherwise happen during a simulation involving wetting and drying.

The overall numerical scheme of the current model is explicit and so its numerical stability is controlled by the Courant-Friedrichs-Lewy (CFL) criterion. For a twodimensional model shallow flow model, the time step for stable simulations may be identified by.

$\Delta t=C \min \left(\min _{i} \frac{\Delta x_{i}}{\left|u_{i}\right|+\sqrt{g h_{i}}}, \min _{i} \frac{\Delta y_{i}}{\left|v_{i}\right|+\sqrt{g h_{i}}}\right)$.
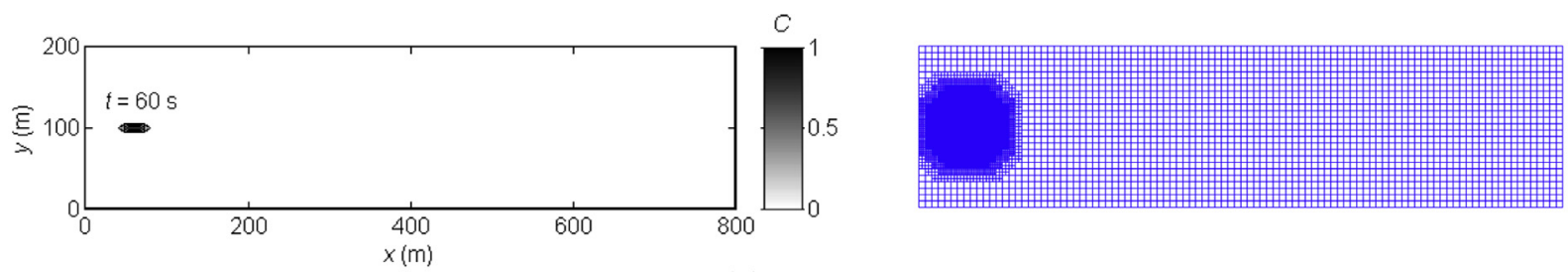

(a) $t=60 \mathrm{~s}$
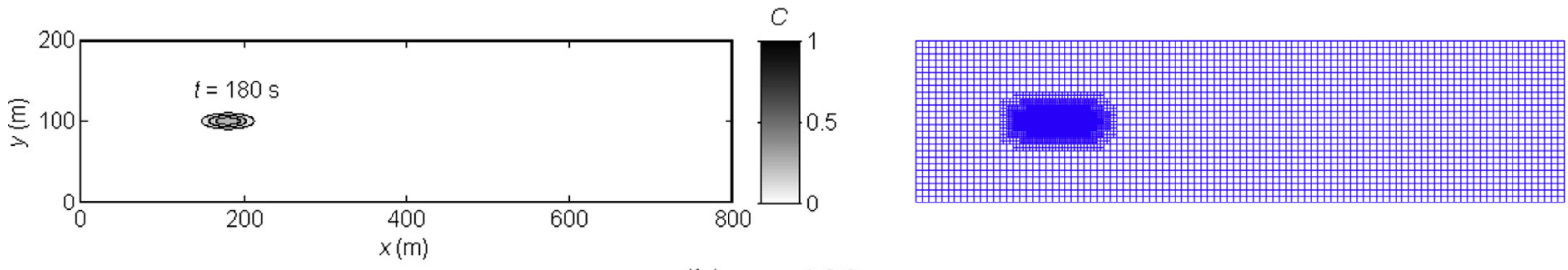

(b) $t=180 \mathrm{~s}$
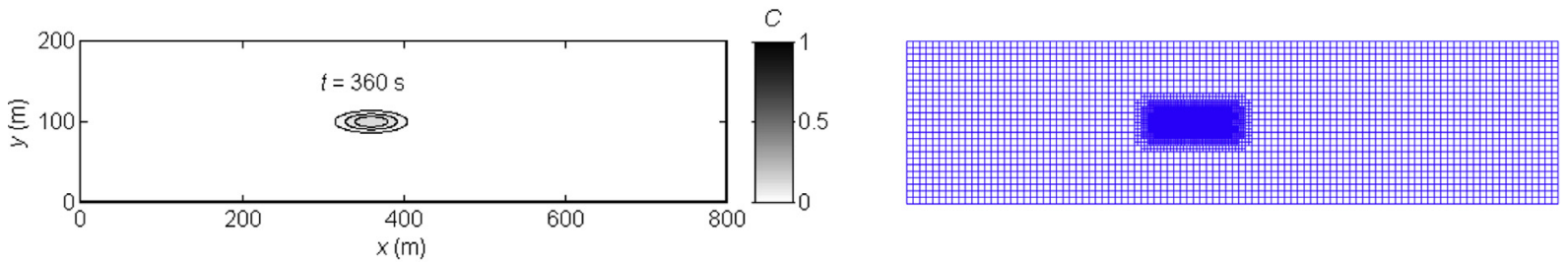

(c) $t=360 \mathrm{~s}$
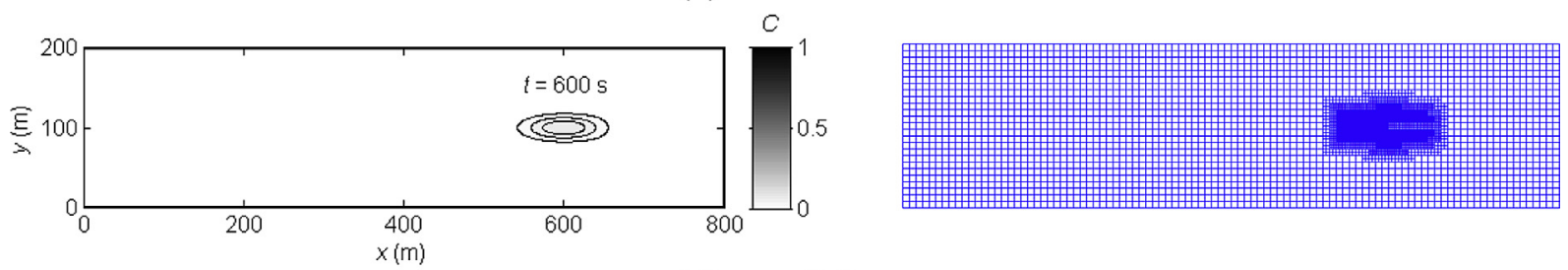

(d) $t=600 \mathrm{~s}$

Fig. 3. Advection and diffusion of a solute cloud: concentration field and adapted grids at (a) $t=60 \mathrm{~s}$; (b) $t=180 \mathrm{~s}$; (c) $t=360 \mathrm{~s}$; (d) $t=600 \mathrm{~s}$. 


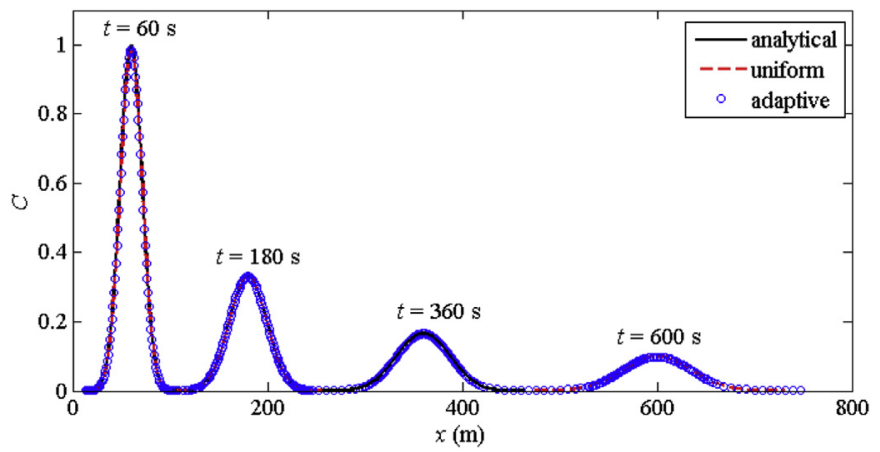

Fig. 4. Advection and diffusion: concentration profiles alone the $x$-direction line, compared with the analytical solution and predictions obtained on corresponding uniform grid.

where $0<C \leq 1$ is the Courant number. The current numerical scheme solves the pre-balanced governing equations incorporated with a local bed modification technique for preserving non-negative water depth and solute concentration for applications involving wetting and drying. This effectively removes the influence of excessive bed slope terms on numerical stability. Therefore, the above CFL condition is implemented to predict the time step for next iteration with $C=0.5$ in all test cases considered in this work for the present 2nd-order numerical scheme. Numerical experiments indicate that inclusion of the extra advection-diffusion equation does not affect the numerical stability in the current model for strong advection test cases as considered in this work.

\section{Adaptive solution}

On an aforementioned structured but non-uniform grid, flow calculation is carried out in the same way as on a uniform grid. As shown in Fig. 2, in order to calculate the $x$-direction face values in cell $i$ on a uniform grid template, flow information is required at nodes $i, e$ and $w$. With flow variables directly available at $i$ and $e$, a uniform grid template can be easily constructed by obtaining the information at $w$ using linear interpolation. Rather than evaluating it directly, $\mathbf{F}_{\boldsymbol{E}}=$ $\left(\mathbf{F}_{\boldsymbol{E} 1}+\mathbf{F}_{\boldsymbol{E} 2}\right) / 2$ is reinforced during flow calculation to maintain mass and momentum conservation for a finite volume scheme.

One of the main advantages of the current model is its capability to allow dynamic grid adaptation, i.e. addition and

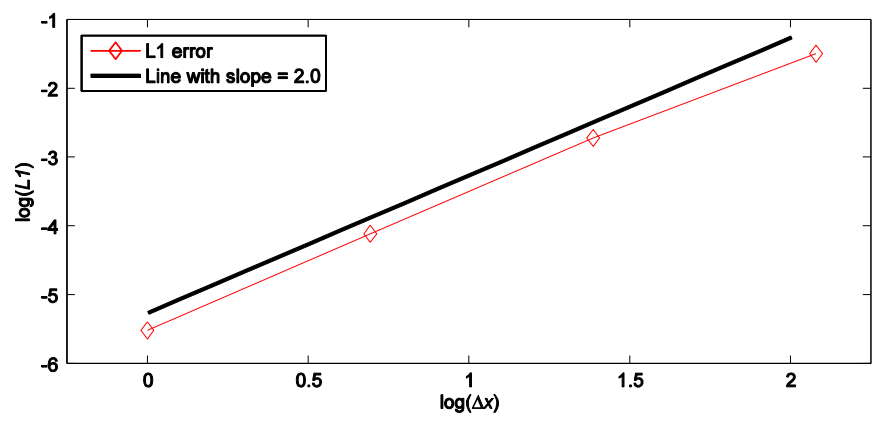

Fig. 5. Advection and diffusion: grid convergence test. removal of grid cells. In order to effectively control the adaption procedure, an error indicator based on the averaged gradients of water level and solution concentration is defined and used in this work. The averaged gradients are calculation by

$G_{\eta_{i}}=\sqrt{(\partial \eta / \partial x)_{i}^{2}+(\partial \eta / \partial y)_{i}^{2}}$

and

$G_{c_{i}}=\sqrt{(\partial c / \partial x)_{i}^{2}+(\partial c / \partial y)_{i}^{2}}$.

Both of them are then normalised as

$\Theta_{\eta i}=G_{\eta i} / P_{q}\left(\mathbf{G}_{\eta}\right)$

and

$\Theta_{c i}=G_{c i} / P_{q}\left(\mathbf{G}_{c}\right)$,

where $\mathbf{G}_{\eta}$ and $\mathbf{G}_{c}$ are the vectors storing corresponding gridbased gradients in the entire computational domain. $P_{q}(\mathbf{X})$ returns the $q$ th percentile of $\mathbf{X}$ with $q=1-s_{a} . s_{a}$ indicates the sensitivity to the error indicator, e.g. $s_{a}=10 \%$ essentially means that $10 \%$ of the flow cells are subject to grid refinement. Finally the error indicator is defined as

$\Theta_{i}=\max \left(\Theta_{\eta i}, \Theta_{c i}\right)$.

Apart from $s_{a}$, another critical value $\Theta_{\text {coar }}$ is also prescribed for grid coarsening. During a simulation, grid adaptation is undertaken at every time step according to the following criteria.

1. If a background cell is not in maximum subdivision and has at least one child with $\Theta_{i}>1$, it will be marked for refinement and its subdivision level becomes $l e v+1$;

2. When all children of a subdivided background cell have $\Theta_{i}>\Theta_{\text {coar }}$, the cell will be marked for coarsening and its subdivision level will be changed to lev -1 .

Independent to the above adaptation procedure, cells are automatically refined to capture moving wet-dry fronts, i.e. a dry cell next to a wet cell will be subdivided to welcome the incoming flow with a high-resolution mesh. Flow variables at the centre of a newly created cell are obtained by linear interpolation.

\section{Results and discussions}

In this section, the current dynamically adaptive grid based solute transport model is applied to simulate three test cases to validate its performance in different aspects. All the simulations run on a Lenovo X61 laptop and $g=9.81 \mathrm{~m} / \mathrm{s}^{2}, s_{a}=0.2$ and $\Theta_{\text {coar }}=0.6$ are used.

\subsection{Advection and diffusion of solute in a uniform flow field}

This first test case features the advection and diffusion of a solute cloud in a steady uniform flow. With $h=1 \mathrm{~m}, u=1 \mathrm{~m} / \mathrm{s}$ 

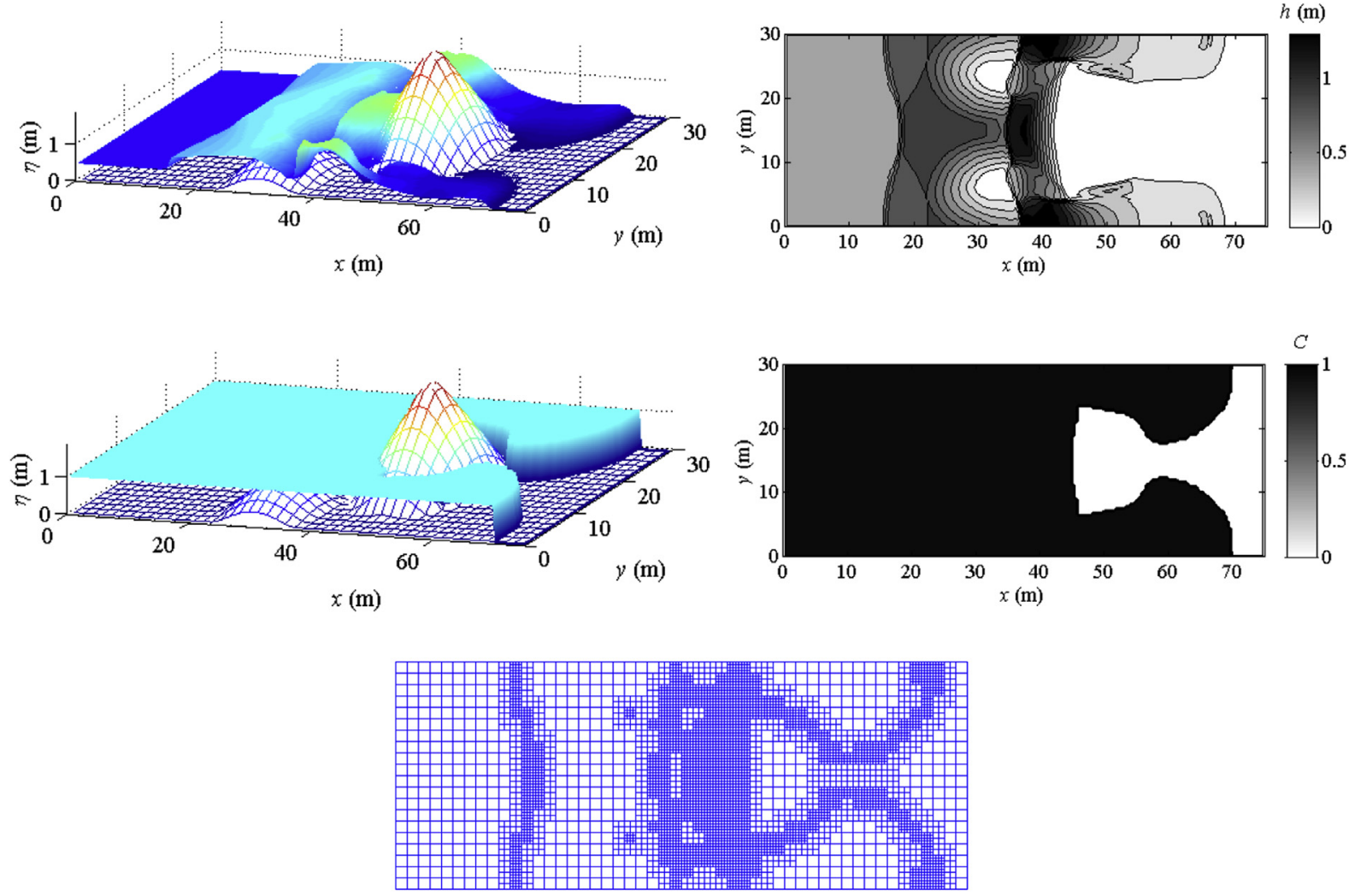

(a) $t=12 \mathrm{~s}$
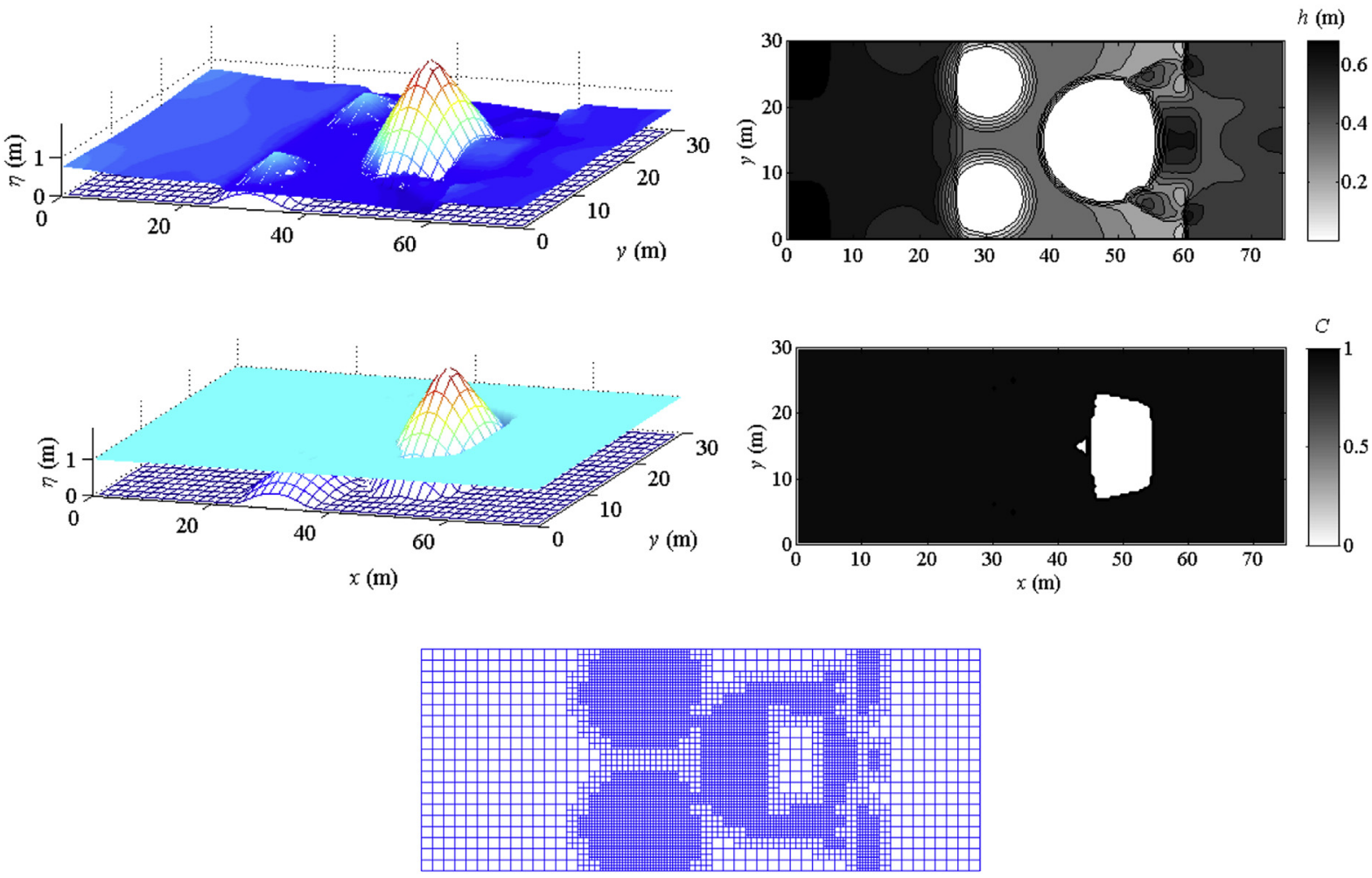

(b) $t=30 \mathrm{~s}$

Fig. 6. Dam break over three humps: numerical predictions at different output times. 


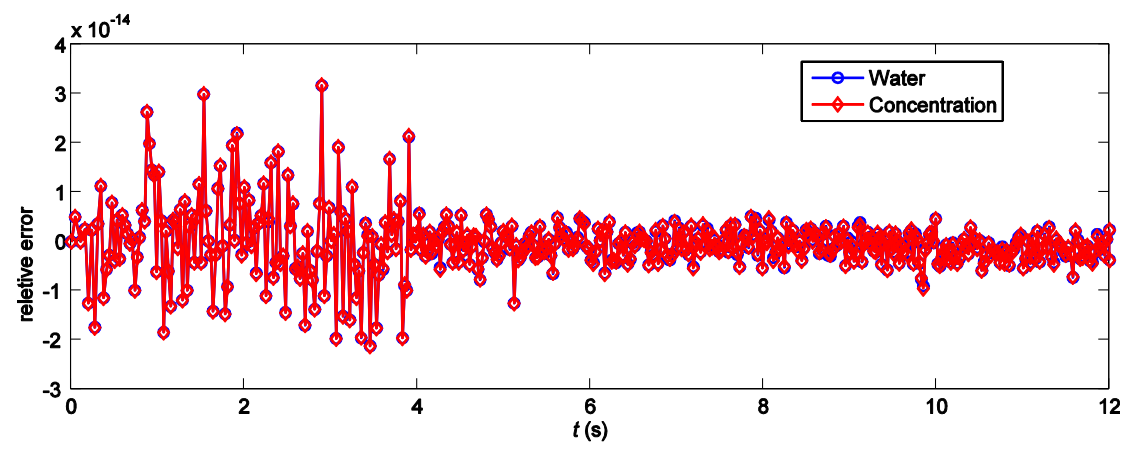

Fig. 7. Dam break over three humps: time histories of mass error for water and solute concentration.

and $v=0$, the background flow takes place in an $800 \mathrm{~m} \times 200 \mathrm{~m}$ frictionless domain with a horizontal bed. The analytical solution of the solute concentration for this idealised case is provided as

$c(x, y)=\frac{C_{0} / h}{4 \pi t \sqrt{D_{x x} D_{y y}}} \exp \left(-\frac{\left(x-x_{0}-u t\right)^{2}}{4 D_{x x} t}-\frac{\left(y-y_{0}\right)^{2}}{4 D_{y y} t}\right)$,

where the basic parametric constants are set to be $C_{0}=233.06, D_{x x}=1.02 \mathrm{~m}^{2} / \mathrm{s}, D_{y y}=0.094 \mathrm{~m}^{2} / \mathrm{s}$, the peak concentration is located at $\left(x_{0}, y_{0}\right)=(0,100)$ at the beginning of the simulation. Simulation starts at $t=60 \mathrm{~s}$ with the initial

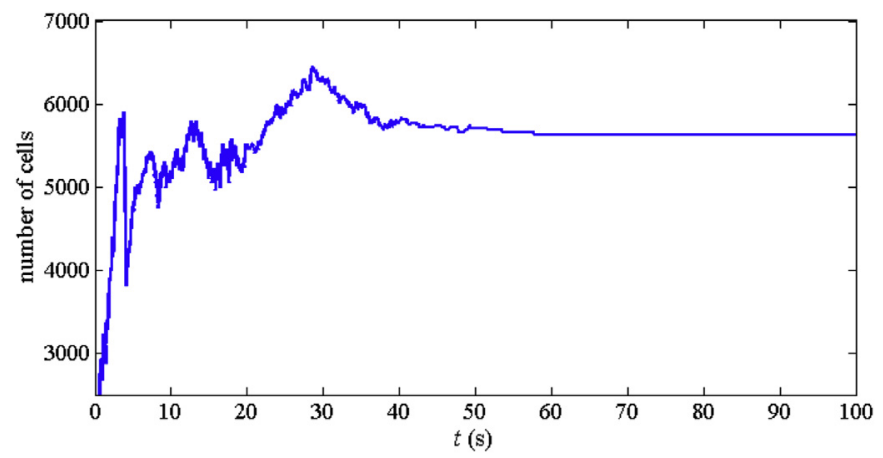

Fig. 8. Dam break over three humps: temporal change of cell population.
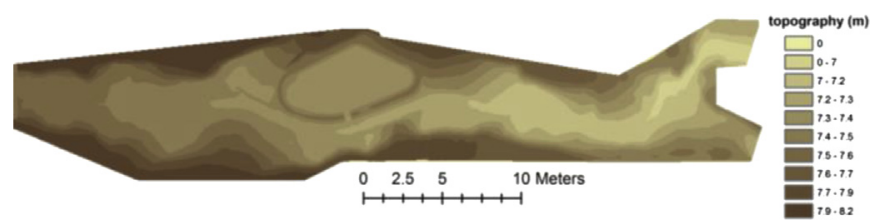

(a)

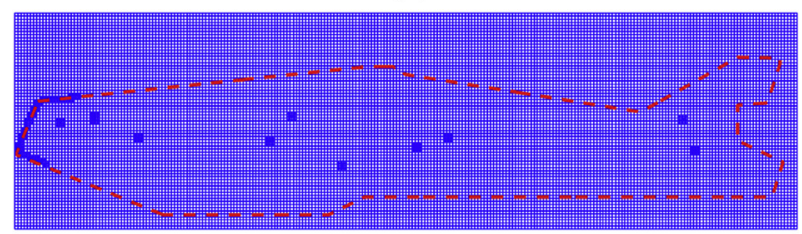

(b)

Fig. 9. Toce River dam break: floodplain. condition of concentration given by (35). The low-resolution background grid consists of $100 \times 25$ cells and is allowed to be refined up to 3 subdivision levels during the simulation, which corresponds to the lowest and highest spatial resolution of $8 \mathrm{~m} \times 8 \mathrm{~m}$ and $1 \mathrm{~m} \times 1 \mathrm{~m}$, respectively. Transimissive conditions are imposed in all four lateral boundaries.

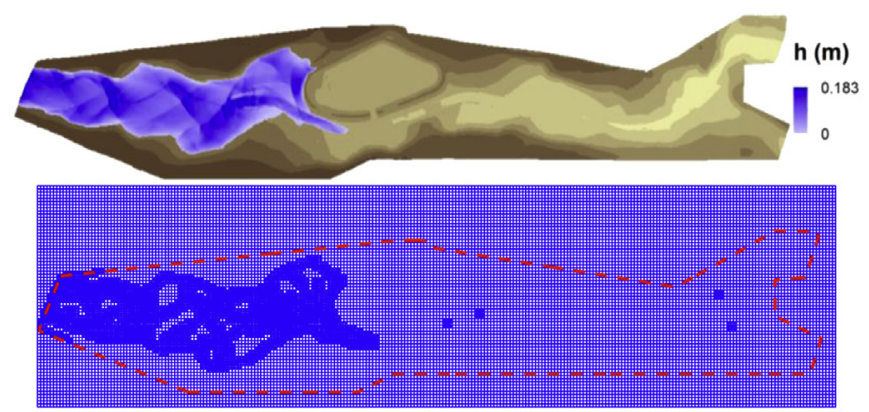

(a) $t=30 \mathrm{~s}$

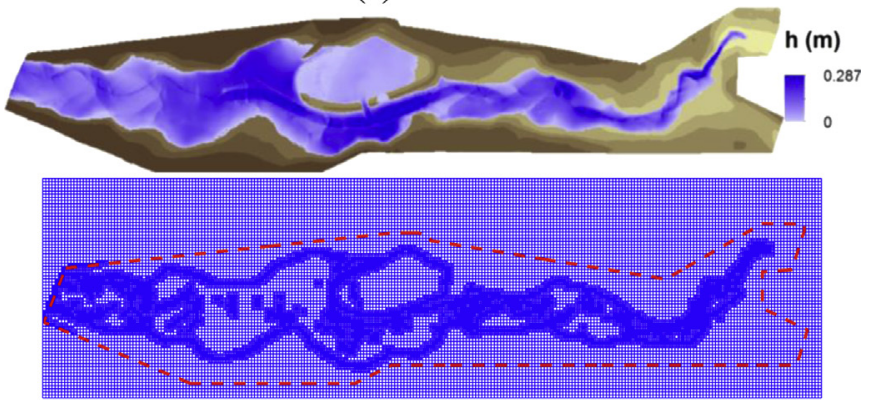

(b) $t=60 \mathrm{~s}$
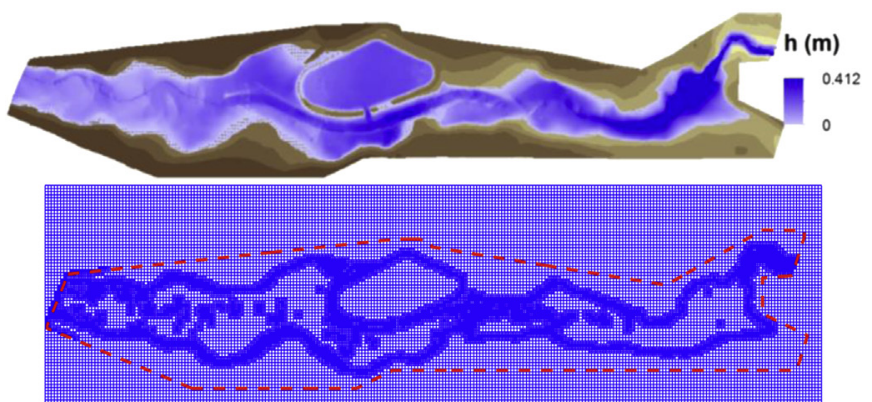

(c) $t=180 \mathrm{~s}$

Fig. 10. Toce River dam break: flood maps and adaptive grids at different output times. 


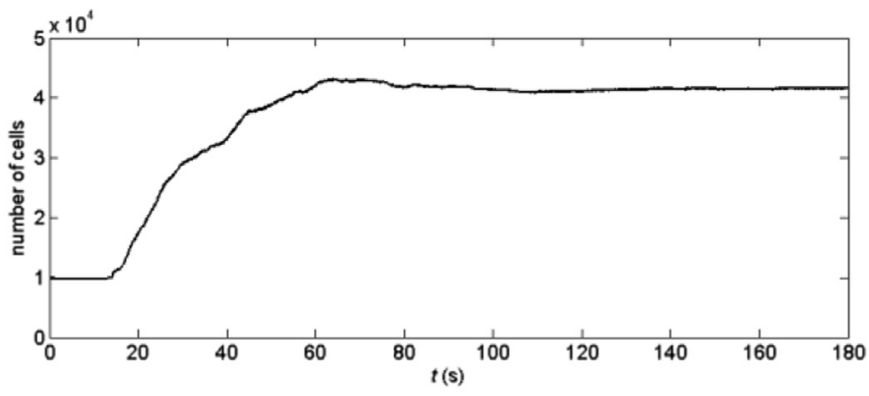

Fig. 11. Toce River dam break: time evolution of cell population.

Simulation is run until $t=600 \mathrm{~s}$ and Fig. 3 presents the numerical results in terms of concentration contours and adapted grids at different output times. It is evident that, starting from $t=60 \mathrm{~s}$, the concentration field is transported to downstream by the flow while undergoing diffusion. The computational grid is observed to adapt to the evolution of the solute concentration throughout the simulation, with refined high-resolution mesh only created to the region with sharp concentration gradients. With coarse mesh used in the rest of the domain, the number of grid cells used in flow calculation is optimised, leading to a substantial saving of computational time without actually compromising the solution accuracy. The numerically predicted profiles of concentration at
Further simulations have also been conducted on uniform grids with $\Delta x=8 \mathrm{~m}, 4 \mathrm{~m}, 2 \mathrm{~m}$ and $1 \mathrm{~m}$ in order to analyse the sensitivity of the numerical solutions to grid resolution. $L_{1}$ error is calculated for solute concentration at the end of each simulation at $t=600 \mathrm{~s}$, which is defined as

$L_{1}=\frac{\sum_{i}\left|c_{i}-\tilde{c}_{i}\right|}{\sum_{i} \tilde{c}_{i}}$,

where $\tilde{c}_{i}$ is the analytical solution of concentration at cell $i$. Fig. 5 plots in double log-scale the $L_{1}$ error against grid resolution $\Delta x$. The result confirms grid convergence for this test and reflects the 2nd-order accuracy of the numerical scheme.

\subsection{Solute advection driven by the dam-break flow over three humps}

Characterised by a rapidly spreading dam-break flow over a complex topography of three humps, this case was used by Liang (2010b) to test the performance of a finite-volume Godunov-type scheme implemented on uniform grids. Herein, this case is reconsidered to demonstrate the advantage of using dynamically adaptive grids. The bottom topography of three humps in a $75 \mathrm{~m} \times 30 \mathrm{~m}$ rectangular close domain is given by

$$
\begin{aligned}
z_{b}(x, y)= & \max \left[0,1-\frac{1}{8} \sqrt{(x-30)^{2}+(y-6)^{2}},\right. \\
& \left.1-\frac{1}{8} \sqrt{(x-30)^{2}+(y-24)^{2}}, 3-\frac{3}{10} \sqrt{(x-47.5)^{2}+(y-15)^{2}}\right] .
\end{aligned}
$$

different output times are illustrated in Fig. 4, in comparison with the analytical solution. The numerical predictions obtained on the adaptive and fine uniform grids are found to be identical for this test, both of which agree perfectly with the analytical solution. However, compared with the $2625.2 \mathrm{~s}$ run time required by the simulation on the fine uniform grid, the adaptive simulation only takes $157.8 \mathrm{~s}$, i.e. the adaptive simulation saves 16.6 times of the computational cost for this test case while providing identical solution.
A dam is installed in a location $16 \mathrm{~m}$ away from the western end to separate the domain into an upstream reservoir and a downstream dry floodplain. The initial water body inside the reservoir is motionless with a constant level of $1.75 \mathrm{~m}$ and polluted by a solute mixture of concentration 1 . The Manning coefficient $n=0.018$ is assumed to be a constant throughout the whole domain. Starting from a coarse background grid of $50 \times 20$ cells, the computational domain is discretized by a mesh that can be refined up to a maximum subdivision level of
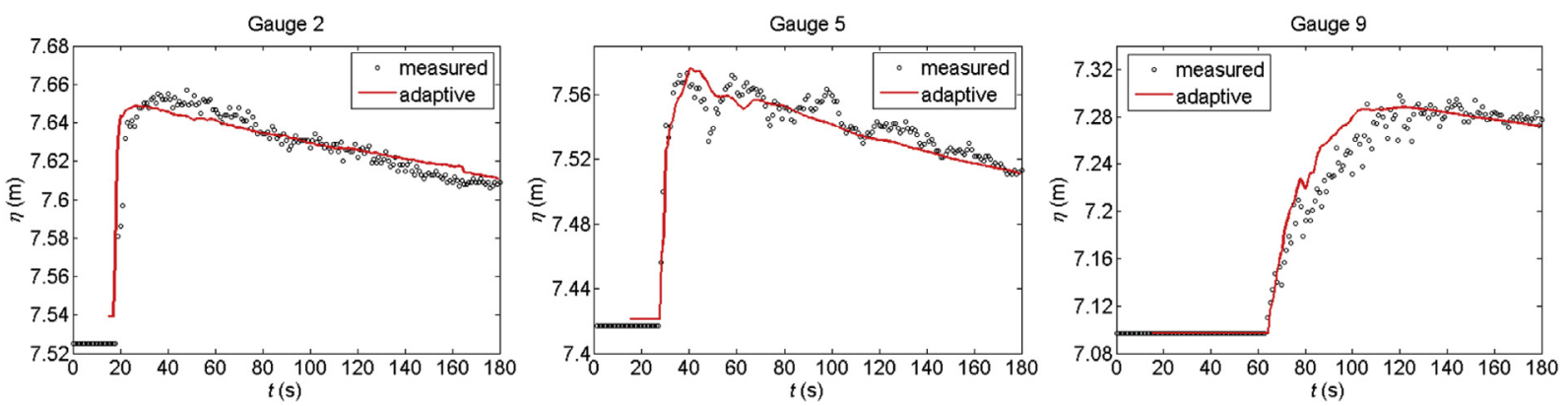

Fig. 12. Toce River dam break: comparison between predicted and measured time histories of water level at different gauge points. 
2. This indicates that the uniform grid of similar resolution will contain $200 \times 80$ cells. The initial non-uniform grid is refined locally in the region representing the dam to give more accurate account of the initial discontinuous water surface. The whole simulation lasts for $300 \mathrm{~s}$.

Fig. 6 presents the numerical predictions at the sample output times of $t=12 \mathrm{~s}$ and $30 \mathrm{~s}$. The Godunov-type scheme is observed to correctly capture the complex flow patterns induced by the dam-break wave (shock) interacting with the three humps and domain boundaries. The numerically predicted wave patterns are identical to those obtained on uniform grids as reported by Liang (2010b). For the solute transport, since there is no diffusion, it is expected that the solution should have concentration of 1 wherever there is water in presence and 0 when the domain is dry. This is obviously the case in the current prediction and no obvious numerical diffusion is detected near the wet-dry front. Fig. 7 presents the relative mass errors calculated for both water and solute concentration. With the maximum error in a scale of $10^{-14}$, the current model apparently ensures mass conservation up to machine precision. The adaptive grid follows the dynamics of the dam-break wave and solute transport to provide highresolution meshes ONLY in those areas with steep gradients of water surface or solute concentration and near the wet-dry front. Fig. 8 shows the temporal change of cell population for the first $100 \mathrm{~s}$ of the whole simulation. On the initial grid, there is a total of 1420 cell. As the dam break occurs and the motions of flow and solute transport become more complex, the number of cells increases and adapts to the flow and solute dynamics at the first stage of the simulation. At about $t=30 \mathrm{~s}$, the number of cells in the computational domain reaches a peak value of about 6400 and gradually converges to a constant of 5632 as the flow gradually settles to become steady. In order to capture the flow features with the same resolution, the uniform grid requires 16,000 cells and consumes $277.3 \mathrm{~s}$ of CPU time to complete the simulation, compared to that of $101 \mathrm{~s}$ on the adaptive grid. Therefore the computation is nearly 3 times more efficient for this more realistic test case and the adaptive grid simulation still represents a significant improvement in computational efficiency while maintaining similar solution accuracy.

\subsection{Point-source solute transport driven by Toce River dam break}

As illustrated in Fig. 9(a), the $55 \mathrm{~m} \times 13 \mathrm{~m}$ physical model of this dam-break test was built in ENEL (Italy) to represent a $5 \mathrm{~km}$ reach of the Toce River in the Northern Alps in Italy. The dam-break flow was triggered by sudden release of flood water from an upstream inflow tank. Temporal change of water level was recorded in 10 gauge stations during the experiment that lasted for $180 \mathrm{~s}$. As part of the experimental dataset, the domain topography was provided on a raster grid with a resolution of $5 \mathrm{~cm}$. A Manning coefficient of $n=0.0162 \mathrm{~s} / \mathrm{m}^{1 / 3}$ was suggested for the entire computation domain.

In the current simulation, the inflow hydrograph is imposed at the western end of the domain and other boundaries are

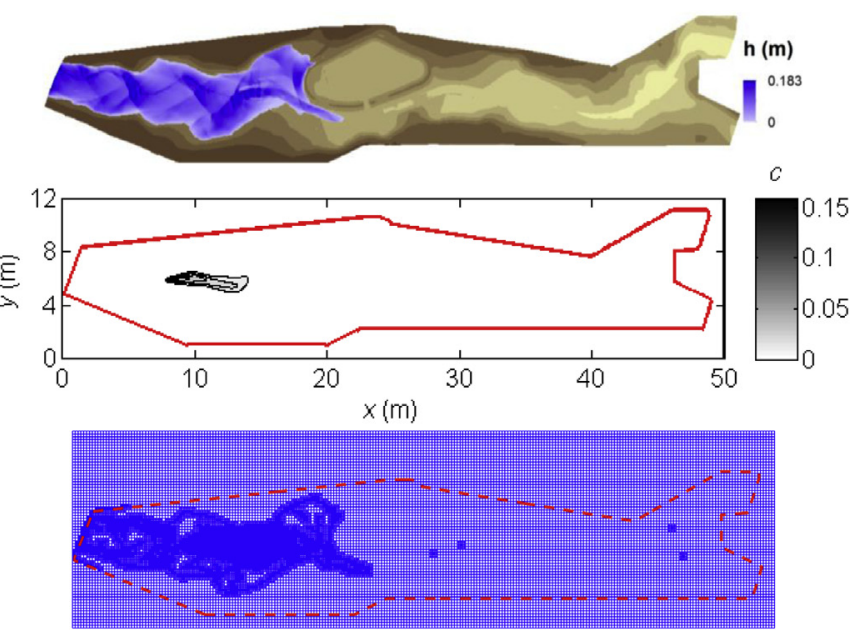

(a) $t=30 \mathrm{~s}$

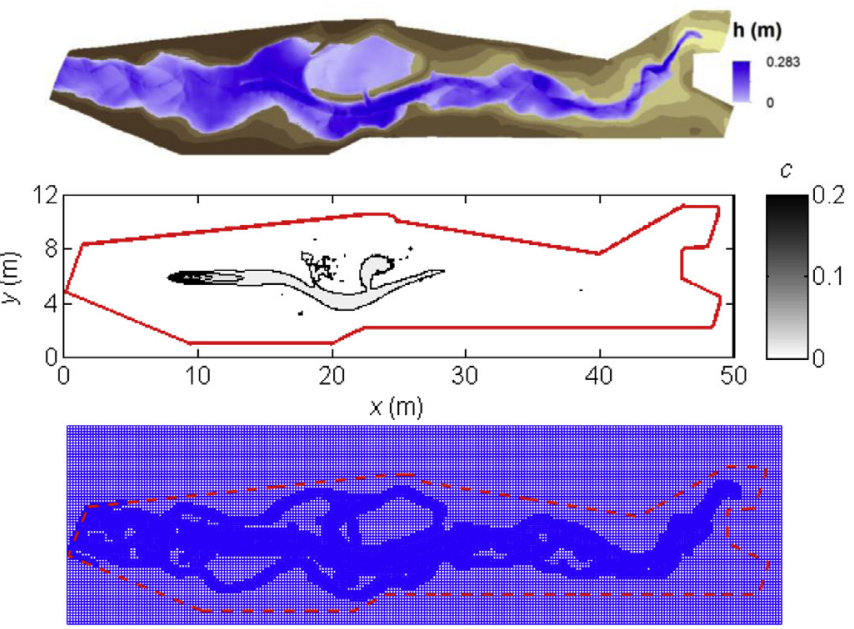

(b) $t=60 \mathrm{~s}$
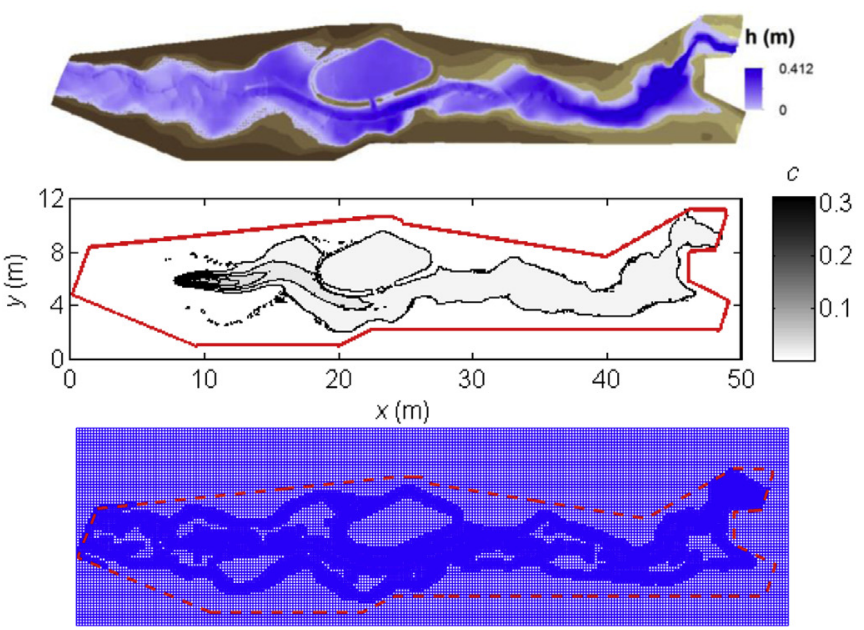

(c) $t=180 \mathrm{~s}$

Fig. 13. Toce River dam break with solution concentration: flood maps, solution concentration contours and adaptive grids at different output times.

assumed to be transmissive. Fig. 9(b) shows the initial grid on which the coarse background grid has $250 \times 60$ cells and the mesh near the inflow boundary is refined up to level 2 to give a resolution at $5 \mathrm{~cm} \times 5 \mathrm{~cm}$ to represent more accurately the 
inlet flow. Refined meshes are also generated to represent the 10 gauge stations. The $180 \mathrm{~s}$ simulation is run for the whole duration of the experiment. A point source given by $s_{1}=1 \mathrm{~m} / \mathrm{s}$ and $c_{s}=1 \mathrm{~kg} / \mathrm{m}^{3}$ is imposed at $(7.868,5.882)$, with the origin of Cartesian coordinate system located at the bottom left corner. The point source of solute concentration is released at $t=25 \mathrm{~s}$ after the simulation starts to ensure the arrival of flood wave front.

In order to validate the model against experimental measurements, the simulation is first carried out without introducing solute to the flow. Fig. 10 presents the predicted flood maps and the associated adapted grids at different output times. The flood flow rushes through the low-lying valley to the downstream after the dam break happens. The flow hydrodynamics is highly influenced by the domain topography and featured by rapid change of wet-dry interface. The grid density dynamically evolves according to the rapid-varying flow hydrodynamics and wet-dry front. The adapted grids as shown in Fig. 10 are able to accurately capture the very complicated flow patterns. Fig. 11 presents the temporal change of cell population, which increases from 9800 cells on the initial grid to a maximum of 43141 after about $64 \mathrm{~s}$ and then reduces slightly to become 41000 for the rest of the simulation. The predicted time histories of water level are compared with the measured data at three sample gauge points in Fig. 12 and very close agreement is achieved. This indicates that the current adaptive grid based model is able to accurately reproduce the complex dam-break flood waves and hence is ready to be used for predicting solution transport driven by highly transient shallow flows.

Fig. 13 provides the model results for the simulation after point-source solution concentration is included, in terms of flood maps, concentration contours and adapted grids at different output times. Due to its overall small magnitude, the point-source inflow does not actually have much effect on the flood hydrodynamics. Driven by the violent dam-bream flow, the solute moves downstream rapidly and becomes mixed with the flood water. At the end of the simulation, the contours of solute concentration are observed to trace out a pattern matching the wet-dry interface, which indicates that a single point-source of pollutant may lead to large-scale contamination and affect water quality in a rapidly varying flood event. The evolution of cell population is presented in Fig. 14, where the number of computational cells increases from that on the

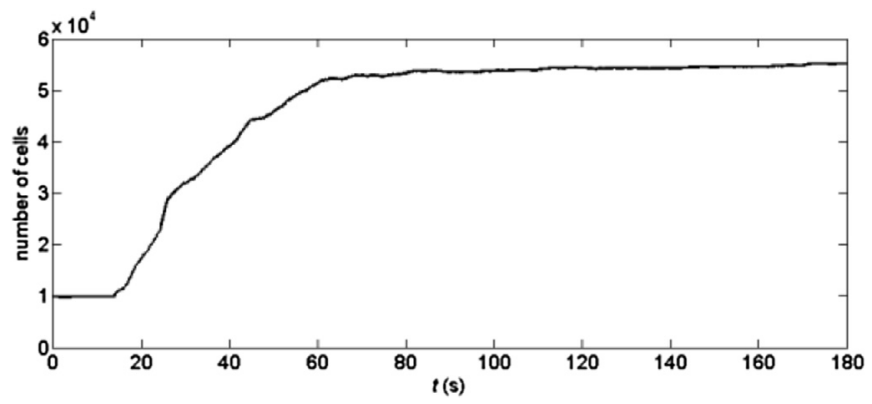

Fig. 14. Toce River dam break with solution concentration: time evolution of cell population. initial grid to a maximum value of 55,000 and then becomes stable. Obviously, the number of cells on the adaptive grid after it becomes stable is much more than that for the simulation without considering solute transport. This indicates that, apart from capturing the complex flow patterns and wet-dry interfaces, adapted mesh is also generated to represent the sharp front of solute concentration. This obviously will lead to more efficient simulation and more accurate results.

\section{Conclusions}

This paper introduces a dynamically adaptive grid based Godunov-type scheme for solving the integrated shallow water and advection-diffusion equations. Compared with uniform grid model as reported by Liang (2010b), the current numerical scheme adopts the MUSCL-Hancock method to achieve second-order accuracy in both space and time and achieves much more efficient simulations without compromising solution accuracy due to the implementation of dynamically adaptive grids. The adaptive mesh simulation starts from a non-uniform grid with local structured property. The initial grid is easy to generate and grid adaptation is straightforward to achieve. Discretization of the governing equations is carried out in an identical way to that on a uniform Cartesian grid after employing a simple linear interpolation scheme. Aiming for practical applications, a non-negative reconstruction technique is implemented to ensure non-negative water depth and solute concentration during a simulation that involves wetting and drying over complex domain topographies.

The current model has been validated against three tests, including the two more realistic cases involving the transport of solute concentration driven by dam-break flood waves. The model is able to represent satisfactorily the flow hydrodynamics in comparison with other numerical predictions reported in literature and experimental measurements. Since the flash flood hydrodynamics shares a number of common features with a dam-break flow, the present model will therefore be directly applicable to more general applications in flash flood modelling and provide a useful tool for assessing the impacts of flash flood on water quality in a complex domain environment.

\section{Acknowledgement}

This work was a result of the following funding projects: (1) 973 Program (Grant no. 2013CB036401); (2) UK EPSRC Global Secure project (EP/K004689/1); and (3) open fund from the Key Laboratory of Water Cycle and Related Land Surface Processes, Institute of Geographic Sciences and Natural Resources Research, Chinese Academy of Sciences (No. WL2011002).

\section{References}

Aricò, C., Sinagra, M., Begnudelli, L., Tucciarelli, T., 2011. MAST-2D diffusive model for flood prediction on domains with triangular delaunay unstructured meshes. Adv. Water Resour. 34 (11), 1427-1449. 
Bates, P.D., De Roo, A.P.J., 2000. A simple raster-based model for flood inundation simulation. J. Hydrol. 236 (1-2), 54-77.

Bates, P.D., Horritt, M.S., Fewtrell, T.J., 2010. A simple inertial formulation of the shallow water equations for efficient two-dimensional flood inundation modelling. J. Hydrol. 387 (1-2), 33-45.

Benkhaldoun, F., Elmahi, I., Seard, M., 2007. Well-balanced finite volume schemes for pollutant transport by shallow water equations on unstructured meshes. J. Comput. Phys. 226 (1), 180-203.

Berger, M.J., Colella, P., 1989. Local adaptive mesh refinement for shock hydrodynamics. J. Comput. Phys. 82 (1), 64-84.

Berger, M.J., George, D.L., LeVeque, R.J., Mandli, K.T., 2011. The GeoClaw software for depth-averaged flows with adaptive refinement. Adv. Water Resour. 34 (9), 1195-1206.

Berger, M.J., Oliger, J., 1984. Adaptive mesh refinement for hyperbolic partial differential equations. J. Comput. Phys. 53 (3), 484-512.

Cea, L., Vázquez-Cendón, M.E., 2012. Unstructured finite volume discretisation of bed friction and convective flux in solute transport models linked to the shallow water equations. J. Comput. Phys. 231 (8), 3317-3339.

George, D.L., 2011. Adaptive finite volume methods with well-balanced Riemann solvers for modeling floods in rugged terrain: application to the Malpasset dam-break flood (France, 1959). Int. J. Numer. Methods Fluids 66 (8), 1000-1018.

Greenberg, J.M., Leroux, A.Y., 1996. A well-balanced scheme for the numerical processing of source terms in hyperbolic equations. SIAM J. Numer. Anal. 33 (1), 1-16.

Kesserwani, G., Liang, Q., 2012. Dynamically adaptive grid based discontinuous Galerkin shallow water model. Adv. Water Resour. 37 (0), 23-39.

Liang, Q., 2010a. Flood simulation using a well-balanced shallow flow model. J. Hydraul. Eng. ASCE 136 (9), 669-675.

Liang, Q., 2010b. A well-balanced and non-negative numerical scheme for solving the integrated shallow water and solute transport equations. Commun. Comput. Phys. 7 (5), 1049-1075.

Liang, Q., 2011. A structured but non-uniform Cartesian grid-based model for the shallow water equations. Int. J. Numer. Methods Fluids 66 (5), $537-554$.

Liang, Q., 2012. A simplified adaptive Cartesian grid system for solving the 2D shallow water equations. Int. J. Numer. Methods Fluids 69 (2), $442-458$.
Liang, Q., Borthwick, A.G.L., 2009. Adaptive quadtree simulation of shallow flows with wet-dry fronts over complex topography. Comput. Fluids 38 (2), 221-234.

Liang, Q., Borthwick, A.G.L., Taylor, P.H., Huang, J., 2003. Godunov-type Quadtree model of species dispersion in shallow flows. In: International Symposium on Shallow Flows. Delft University of Technology, The Netherlands.

Murillo, J., Burguete, J., Brufau, P., Carcía-Navarro, P., 2005. Coupling between shallow water and solute flow equations: analysis and management of source terms in 2D. Int. J. Numer. Methods Fluids 49 (3), 267-299.

Murillo, J., Carcía-Navarro, P., Burguete, J., Brufau, P., 2006. A conservative 2D model of inundation flow with solute transport over dry bed. Int. J. Numer. Methods Fluids 52 (10), 1059-1092.

Murillo, J., García-Navarro, P., 2011. Improved Riemann solvers for complex transport in two-dimensional unsteady shallow flow. J. Comput. Phys. 230 (19), 7202-7239.

Murillo, J., García-Navarro, P., Burguete, J., 2008. Analysis of a second-order upwind method for the simulation of solute transport in 2D shallow water flow. Int. J. Numer. Methods Fluids 56 (6), 661-686.

Murillo, J., García-Navarro, P., Burguete, J., 2009. Conservative numerical simulation of multi-component transport in two-dimensional unsteady shallow water flow. J. Comput. Phys. 228 (15), 5539-5573.

Petti, M., Bosa, S., 2007. Accurate shock-capturing finite volume method for advection-dominated flow and pollution transport. Comput. Fluids 36 (2), $455-466$.

Popinet, S., 2011. Quadtree-adaptive tsunami modelling. Ocean. Dyn. 61 (9), 1261-1285.

Roger, B., Fujihara, M., Borthwick, A.G.L., 2001. Adaptive Q-tree Godunovtype scheme for shallow water equations. Int. J. Numer. Methods Fluids 35 (3), 247-280.

Toro, E.F., 2001. Shock-capturing Methods for Free-surface Shallow Flows. John Wiley \& Son, Chichester.

Wang, J.P., Liang, Q., 2011. Testing a new adaptive grid-based shallow flow model for different types of flood simulations. J. Flood Risk Manag. 4 (2), 96-103.

Wang, Y., Liang, Q., Kesserwani, G., Hall, J.W., 2011. A positivity-preserving zero-inertia model for flood simulation. Comput. Fluids 46 (1), 505-511.

Zhang, C., Liang, Q., Yin, J., 2013. A first-order adaptive solution to rapidly spreading flood waves. Prog. Comput. Fluid Dyn. 13 (1), 1-10. 